\title{
An In Vitro Co-culture Mouse Model Demonstrates Efficient Vaccine-Mediated Control of Francisella tularensis SCHU S4 and Identifies Nitric Oxide as a Predictor of Efficacy
}

\author{
Igor Golovliov ${ }^{1}$, Helena Lindgren ${ }^{1}$, Kjell Eneslätt ${ }^{1}$, Wayne Conlan ${ }^{2}$, Amandine Mosnier ${ }^{3}$, \\ Thomas Henry ${ }^{3}$ and Anders Sjöstedt ${ }^{1 *}$ \\ 1 Laboratory for Molecular Infection Medicine Sweden, Department of Clinical Microbiology, Clinical Bacteriology, and Umeå \\ University, Umeå, Sweden, ${ }^{2}$ National Research Council Canada, Institute for Biological Sciences, Ottawa, ON, Canada, \\ ${ }^{3}$ Centre International de Recherche en Infectiologie, Institut national de la santé et de la recherche médicale, U1111, Lyon, \\ France
}

\section{OPEN ACCESS}

Edited by:

Alfredo G. Torres,

University of Texas Medical Branch,

USA

Reviewed by:

Tonyia Eaves-Pyles,

University of Texas Medical Branch

USA

Cornelia Blume,

University of Southampton, UK

*Correspondence:

Anders Sjösted

anders.sjostedt@umu.se

Received: 10 September 2016 Accepted: 01 November 2016 Published: 25 November 2016

Citation:

Golovliov I, Lindgren H, Eneslätt K, Conlan W, Mosnier A, Henry T and

Sjöstedt A (2016) An In Vitro

Co-culture Mouse Model

Demonstrates Efficient

Vaccine-Mediated Control of Francisella tularensis SCHU S4 and Identifies Nitric Oxide as a Predictor of

Efficacy.

Front. Cell. Infect. Microbiol. 6:152. doi: 10.3389/fcimb.2016.00152
Francisella tularensis is a highly virulent intracellular bacterium and cell-mediated immunity is critical for protection, but mechanisms of protection against highly virulent variants, such as the prototypic strain F. tularensis strain SCHU S4, are poorly understood. To this end, we established a co-culture system, based on splenocytes from naïve, or immunized mice and in vitro infected bone marrow-derived macrophages that allowed assessment of mechanisms controlling infection with F. tularensis. We utilized the system to understand why the $c / p B$ gene deletion mutant, $\triangle c / p B$, of SCHU S4 shows superior efficacy as a vaccine in the mouse model as compared to the existing human vaccine, the live vaccine strain (LVS). Compared to naïve splenocytes, $\Delta c / p B-$, or LVS-immune splenocytes conferred very significant control of a SCHU S4 infection and the $\triangle c / p B$-immune splenocytes were superior to the LVS-immune splenocytes. Cultures with the $\triangle c / p B$-immune splenocytes also contained higher levels of IFN- $\gamma$, $\mathrm{IL}-17$, and GM-CSF and nitric oxide, and T cells expressing combinations of IFN- $\gamma$, TNF- $\alpha$, and IL-17, than did cultures with LVS-immune splenocytes. There was strong inverse correlation between bacterial replication and levels of nitrite, an end product of nitric oxide, and essentially no control was observed when BMDM from iNOS ${ }^{-/-}$mice were infected. Collectively, the co-culture model identified a critical role of nitric oxide for protection against a highly virulent strain of $F$. tularensis.

Keywords: F. tularensis SCHU S4, in vitro co-culture model, mouse immune response, correlates of protection

\section{INTRODUCTION}

Francisella tularensis is a highly virulent facultative intracellular bacterium causing the severe disease tularemia in many mammalian species (Sjöstedt, 2007). Two subspecies are common human pathogens, subspecies tularensis (type A), which causes disease with high mortality if untreated, and the less aggressive subspecies holarctica (type B), which despite its lower virulence 
may cause serious illness in humans. Tularemia is widespread over the Northern hemisphere and a significant health problem, in particular in certain parts of Scandinavia, in parts of Eastern Europe, and in Turkey, but a rather uncommon disease in most other parts of the world. The live vaccine strain (LVS) is a human vaccine strain, which confers efficacious protection against laboratory-acquired infection, as demonstrated by the fact that the incidence of tularemia among the laboratory staff decreased by $95 \%$ after its introduction (Burke, 1977). Despite this success, studies on volunteers in the 1960s revealed that it did not confer efficacious protection against aerosol infection [reviewed by Conlan et al. (Conlan, 2011)]. Therefore, there is a need for development of more efficacious Francisella vaccines and previously, we analyzed if defined mutants of SCHU S4 (type A) could serve as such vaccine candidates and identified that the $\Delta c l p B$ mutant conferred superior efficacy compared to LVS, despite that the former was more attenuated (Kadzhaev et al., 2009; Conlan et al., 2010; Ryden et al., 2012). It encodes an AAA + chaperone, although the exact function in F. tularensis is still unknown.

Due to the essential role of CMI for host protection against tularemia, a thorough understanding of its characteristics will be necessary to identify how the infection is controlled. There is accumulating evidence that the protective mechanisms are effectuated via a complex interplay of multiple $\mathrm{T}$ cell subsets and other immune mechanisms, and not a single specific immune activity (Elkins et al., 2007; De Pascalis et al., 2008, 2012, 2014; Cowley and Elkins, 2011; Eneslätt et al., 2011, 2012; Mahawar et al., 2013; Griffin et al., 2015). Thus, the mechanisms cannot be delineated using simple proliferation assays but will require assays that closely mimic the in vivo situation. Therefore, new models are needed to better understand protective mechanisms and validate potential correlates of protective immunity. In this regard, a functional in vitro splenocyte-bone marrow-derived macrophage (BMDM) co-culture assay for measuring potential correlates of protection for tularemia vaccines was developed and substantial work has been performed with the aim to establish correlates of protection against $F$. tularensis (Cowley and Elkins, 2003; Cowley et al., 2005; Collazo et al., 2009; Elkins et al., 2011; De Pascalis et al., 2012, 2014; Mahawar et al., 2013; Griffin et al., 2015). Pascalis et al. showed that the greater efficacy of LVS vs. attenuated derivatives correlated with relative bactericidal activity in the co-culture system (De Pascalis et al., 2012). The relevance of such co-culture assays has to some extent been validated by the demonstration that the identified immune molecules play important roles in vivo (Kurtz et al., 2013; Melillo et al., 2013, 2014). A limitation of the published work using the splenocyte-BMDM co-culture model has been the extensive use of the attenuated LVS strain and there are very few studies, which have utilized fully virulent F. tularensis (Mahawar et al., 2013; Griffin et al., 2015).

The absence of any demonstrable correlate(s) of protection is an obstacle to the licensure of $F$. tularensis vaccines. Tularemia is in most countries an unusual disease and even in endemic areas, it appears with very irregular intervals (Sjöstedt, 2007). Therefore, efficacy in a vaccinated population will not be possible to evaluate. Moreover, although challenge studies of volunteers were performed in the 1950s (Saslaw et al., 1961a,b), such studies, in view of the severity of respiratory tularemia, are very unlikely to be approved today. Therefore, a tularemia vaccine, as well as other vaccines against unusual, aggressive diseases, need to be evaluated according to the FDA Animal Rule (Snoy, 2010). The regulation stipulates that efficacy testing can be performed exclusively using animal models, provided that the mechanisms of action of the vaccine are sufficiently well characterized to permit extrapolation of efficacy to humans. Only one vaccine has so far been approved according to the Animal Rule (US. Food Drug Administration., 2015).

Efficacy of experimental tularemia vaccines has been demonstrated using mouse, rat, rabbit, and non-human primate models, but none of these have been approved by FDA. Thus, models to test tularemia vaccine efficacy will require further characterization before they can be approved, e.g., the identification of correlates of immunity and protection. This would also include models that would not necessitate lethal animal challenges. Therefore, we wanted to investigate if a coculture assay based on infection of mouse bone marrow-derived macrophages could be utilized to assess vaccine efficacy against the highly virulent SCHU S4 strain. In view of the previously described superior vaccine-mediated protection conferred by the vaccine candidate $\Delta c l p B$ compared to LVS (Kadzhaev et al., 2009; Conlan et al., 2010; Ryden et al., 2012), we asked if this superior trait also was reflected using the co-culture assay. Indeed, we observed that $\triangle c l p B$-immune splenocytes conferred superior control of the SCHU S4 strain compared to LVS-immune splenocytes and efficacy strongly correlated with levels of nitric oxide.

\section{MATERIALS AND METHODS}

\section{Bacterial Strains}

F. tularensis LVS was originally obtained from the American Type Culture Collection (ATCC 29684). F. tularensis strain SCHU S4 (F. tularensis subsp. tularensis) was obtained from the Francisella Strain Collection of the Swedish Defense Research Agency, Umeå, Sweden. The generation in our laboratory of the $\Delta c l p B$ strain by allelic replacement of the $c l p \mathrm{~B}$ gene, a procedure that did not introduce any recombinant DNA in the strain, has been described previously (Conlan et al., 2010). All bacteriological work related to the SCHU S4 strain was carried out in a biosafety level 3 facility certified by the Swedish Work Environment Authority.

\section{Animals}

In the experiments, Balb/c or C57/BL6 mice obtained from Charles River, Germany were used. When required, mice were immunized with a dose of approximately $5 \times 10^{3} \mathrm{CFU}$ of the LVS or the $\triangle c l p B$ strain subcutaneously. The ensuing infection resulted in no or very mild objective symptoms between days 4 and 6 of the infection. Ethical approval for all of the described mouse experiments was obtained from the Ethical Committee on Animal Research, Umeå, Sweden, A99-11, and A67-14 and the University of Lyon, France (CECCAPP) under the protocol number \#ENS_2012_061. 


\section{Generation of BMDM}

Bone marrow was flushed from femurs of Balb/c or C57BL6/J mice with Dulbecco's Modified Eagle Medium (DMEM). Typically, femurs from two mice were used in each experiment. Cells were washed, a single-cell suspension was prepared by gentle pipetting in complete DMEM [DMEM supplemented with $10 \%$ of heat-inactivated fetal bovine serum, 0.2 of $\mathrm{mM}$ L-glutamine, (Life Technologies), $1 \mathrm{mM}$ of HEPES buffer (Life Technologies), and $50 \mu \mathrm{M}$ of $\beta$-mercaptoethanol), and $10 \%$ of L-929 conditioned medium. After counting, $5 \times 10^{6}$ BMDM in $10 \mathrm{ml}$ of DMEM were added to a $10 \mathrm{~cm}$ Petri dish and incubated at $37^{\circ} \mathrm{C}$ and $5 \% \mathrm{CO}_{2}$. After 3 days, $5 \mathrm{ml}$ of complete DMEM containing L-929 conditioned medium were added. After 7 days of total incubation, medium was removed, $10 \mathrm{ml}$ of cold PBS/10 mM EDTA was added and the dishes were incubated on ice for $30 \mathrm{~min}$. The macrophages were carefully collected by pipetting, centrifuged, resuspended in complete DMEM and 5 $\times 10^{5}$ cells per well were added to 24-well plates and incubated overnight at $37^{\circ} \mathrm{C}$ and $5 \% \mathrm{CO}_{2}$ and then used in the co-culture assay. The number of viable BMDM was determined after trypan blue staining using Vi-CELL XR cell viability analyzer (Beckman Coulter).

\section{Splenocyte Preparation}

Four to 5 weeks following immunization of Balb/c or C57/BL6 mice, spleens were aseptically removed from mice and cells were released by gently squeezing the organs with an L-shaped needle. Splenocytes from three mice were used for each group. Splenocytes were prepared essentially as described (Bosio and Elkins, 2001). A single-cell suspension was prepared, centrifuged and erythrocytes were lysed with ammonium chloride. Cells were washed by centrifugation with PBS $+2 \%$ FBS, and resuspended finally in complete DMEM. The number of viable splenocytes was determined after trypan blue staining using Vi-CELL XR cell viability analyzer (Beckman Coulter). The splenocytes were then used either in the co-culture assay or in the in vitro recall response assay.

\section{Infection of the BMDM in the Co-culture Assay}

Bacteria were grown overnight on Gc-agar plates, resuspended in PBS, and added to the BMDM monolayer at an MOI of 1:5 (bacteria:BMDM). After uptake for $2 \mathrm{~h}$, medium was removed and the macrophage monolayer was washed twice using sterile PBS at RT. One $\mathrm{ml}$ of cDMEM containing $20 \mu \mathrm{g} / \mathrm{ml}$ gentamicin was added to each well, plates were incubated for an additional $45 \mathrm{~min}$ and washed twice with PBS. Following the last wash, each BMDM monolayer was overlaid with $1 \mathrm{ml}$ of complete DMEM with $2.5 \times 10^{6}$ of congenic splenocytes, giving a ratio BMDM:splenocytes of 1:5. Bacterial counts were determined by lysis of the cells and plating of serial dilutions (Golovliov et al., 2003). In indicated cultures, $1 \mathrm{mM}$ of $\mathrm{N}^{\mathrm{G}}$ monomethyl-L-arginine (NMMLA) was added simultaneously with the splenocytes and maintained for the remainder of the experiment.

\section{Optimization of the in vitro Splenocyte-BMDM Co-culture Model}

The in vitro co-culture assay was previously established for the LVS strain (Bosio and Elkins, 2001; Cowley and Elkins, 2003). We now optimized the assay so it reproducibly could be used for the SCHU S4 strain as well. This involved the optimization of the ratio between T-cells with the antigen-presenting cells. It was found that a minimum of $4 \times 10^{5} \mathrm{BMDM}$ per well and 5fold more immune splenocytes, i.e., a ratio of 1:5, resulted in the best control of both intracellular LVS and SCHU S4. When the ratio was 1:1, the growth inhibition of LVS and SCHU S4 was on average $0.7 \pm 0.1$ and $1.3 \pm 0.4 \log _{10} \mathrm{CFU}$, respectively, less than the growth inhibition using the ratio of 1:5. A ratio of 1:10 led to results similar to those using the 1:5 ratio. Also, the role of the bacterial MOI was investigated and bacterial uptake was determined by lysis of the cultures after addition of bacteria for $120 \mathrm{~min}$ and it was determined that an MOI of 1:5 (bacteria/BMDM) resulted in optimal growth inhibition.

\section{In vitro Recall Response and Lymphocyte Proliferation Assay}

Splenocytes were prepared from naïve mice or from mice immunized with LVS or $\triangle c l p \mathrm{~B}$ as described above and seeded at $2 \times 10^{5}$ in $200 \mu \mathrm{L}$ of complete DMEM supplemented with 50 $\mu \mathrm{M}$ of $\beta$-mercaptoethanol per well in 96 -well plates. Splenocytes were stimulated with a 1:1 mix of formalin-fixed LVS (ffLVS) and formalin-fixed SCHU S4 (ffSCHUS4) antigen at a final concentration of 0.5 bacteria/splenocyte or without antigen and incubated in a humidified atmosphere with $5 \% \mathrm{CO}_{2}$ at $37^{\circ} \mathrm{C}$. After 3 days the proliferative response of the splenocytes was detected by measuring the $\left[{ }^{3} \mathrm{H}\right]$-thymidine incorporation as previously described (Ericsson et al., 1994) or analyzed by FACS as described below.

\section{Nitrite Measurement}

The amount of $\mathrm{NO}_{2}^{-}$in the culture supernatants after $72 \mathrm{~h}$ incubation was determined by use of the Griess reagent (Giovannoni et al., 1997). One hundred microliter of the culture supernatant was mixed with $100 \mu \mathrm{l}$ each of the Griess reagents, p-Aminobenzenesulfonamide (58 $\mathrm{mM}$ in $\left.5 \% \mathrm{H}_{3} \mathrm{PO}_{4}\right)$, and 2,6,8Trihydroxypurine (3.9 mM) (Sigma). After 10 min of incubation at RT, the absorbance at $540 \mathrm{~nm}$ was recorded. The concentration of $\mathrm{NO}_{2}^{-}$was determined by preparing a standard curve of sodium nitrite. The lower limit of detection in the assay was $1.0 \mu \mathrm{M}$, i.e., below that concentration, medium spiked with nitrite showed an absorbance indistinguishable from that of buffer alone.

\section{Multiplex Cytokine Analysis}

Cell culture supernatants, $50 \mu \mathrm{L} /$ well, were collected from the same cell cultures as used for assessment of intracellular bacterial replication and stored frozen at $-80^{\circ} \mathrm{C}$ until analyzed using a commercial 23-plex kit (catalog \#M60009RDPD) or a custommade 9-plex kit according to the manufacturer's instructions with a Bio-Plex 200 system (BioRad Laboratories Inc, Hercules, CA, USA). The 9-plex kit contained following cytokines: IL-2, IL-6, IL-12p40, IL-17, IFN- $\gamma$, MIP-1 $\beta$, GM-CSF, RANTES, and TNF- $\alpha$. 


\section{Flow Cytometry Analysis of Surface Markers and Intracellular Cytokine Staining}

Cells were collected after $72 \mathrm{~h}$ of incubation from either the lymphocyte proliferation assay or from the co-culture assay. Non-adherent cells were transferred to a new plate and 5 $\mu \mathrm{g} / \mathrm{mL}$ of Brefeldin A was added. Four h later, plates were centrifuged for $3 \mathrm{~min}$ at $500 \times \mathrm{g}$ and supernatants were removed. After blocking of the Fc $\gamma$ receptors with CD16/CD32 mouse BD Fc block (clone 2.4G2, BD Biosciences), cells were labeled with cell surface marker monoclonal antibodies (mAb) and conjugated intracellular cytokine $\mathrm{mAb}$ as recommended by $\mathrm{BD}$ Biosciences. The following $\mathrm{mAb}$ conjugates were used: $\mathrm{CD} 3-$ APCCy7 (clone 17A2, BD Biosciences), CD4-AF700 (clone RM4-5, BD Biosciences), CD8-PerCPCy5.5 (clone 53-6.7, BD Biosciences), IFN $\gamma$-PE-CF594 (clone XMG1.2, BD Biosciences), TNF- $\alpha$-Brilliant violet 421 (clone MP6-XT22, BioLegend), IL17A-PE/Cy7 (clone TC11-18H10.1, BD Biosciences). Aqua Viability Dye (Molecular Probes/Invitrogen) was added to distinguish live and dead cells. Cells were acquired using an LSRII flow cytometer (BD Biosciences) with FACSDiva software (BD Biosciences). Results were analyzed using FlowJo software (Tree Star).

\section{Data Analysis and Statistical Methods}

Two sample 2-tailed $t$-test, Mann-Whitney $U$ or for paired data, Wilcoxon's signed rank test, were used to identify significant differences $(P<0.05)$ between data sets. To analyze correlation between data sets, Spearman's rank correlation test was used. A correlation with a coefficient (RS) above 0.4 was considered to demonstrate significant correlation, and a coefficient above 0.7 was considered to indicate strong correlation. When multiple experiments formed the basis for the analysis, the cumulative data were presented as boxplots. In each boxplot, the line through each box shows the median, with quartile one, and three as the lower and upper limits of each box. The end of the vertical lines indicates maximum and minimum values, respectively.

\section{RESULTS}

\section{Splenocyte Recall Response}

Splenocytes from mice immunized with LVS or $\Delta c l p \mathrm{~B}$ were stimulated with formalin-killed $F$. tularensis antigen in vitro to measure the recall response of the $\mathrm{T}$-cells with regard to proliferation and expression of cytokines. To accommodate for the different backgrounds of the two strains, a mixture prepared of equal amounts from LVS and SCHU S4 was analyzed to assess the immune reactivity. Both LVS- and $\Delta c l p B$-immune $\mathrm{Balb} / \mathrm{c}$ splenocytes responded with very prominent proliferative responses to the antigen and the responses were stronger, although not significantly stronger, of $\Delta c l p \mathrm{~B}$ - compared to LVS-immune splenocytes $(P=0.16$; Figure 1A). Intracellular staining for IFN- $\gamma$ and TNF- $\alpha$ showed that the percentages of $\mathrm{CD}^{+}{ }^{+}$and $\mathrm{CD} 8{ }^{+} \mathrm{T}$-cells expressing the respective cytokine were significantly higher $(P<0.05)$ among $\Delta c l p \mathrm{~B}$ - than LVS-immune splenocytes (Figures 1B-E).

Overall, the results show that $\Delta c l p \mathrm{~B}$-immune splenocytes demonstrated more prominent recall responses in vitro to the $F$. tularensis antigen than did LVS-immune splenocytes.

\section{Growth Inhibition of $F$. tularensis LVS and Cytokine Production Conferred by LVS- or $\Delta c / p B-I m m u n e$ Splenocytes}

The capacity of splenocytes from LVS- or $\Delta c l p B$-immunized mice to inhibit the intracellular growth of LVS in splenocyteBMDM co-cultures was assessed. Addition of either type of immune splenocyte to the cultures significantly inhibited growth of LVS better than did naïve splenocytes, the median growth and the interquartile (IQ) one and three for all experiments performed were $3.2[2.4,3.8]$ and $2.4[1.7,3.4] \log _{10} \mathrm{CFU}$ for LVS-immune splenocytes and $\Delta c l p \mathrm{~B}$-immune splenocytes, respectively, vs. $5.2[4.9,5.6] \log _{10} \mathrm{CFU}$ for naïve splenocytes $(P$ $<0.001$ for immune $v s$. naïve). The $\Delta c l p B$-immune splenocytes showed superior control compared to LVS-immune splenocytes, $P<0.001$ (Figure 2A). Not only growth inhibition was more prominent in cultures with $\Delta c l p \mathrm{~B}$-immune than LVS-immune splenocytes, but also levels of IFN- $\gamma$, IL-17, and GM-CSF in the culture supernatants were higher $(P<0.001 ; P<0.05$ and $P<$ 0.001, respectively; Figures 2B-D).

In total, levels of 23 cytokines were analyzed in the supernatants from the co-cultures and 17 of those were found to be upregulated more than 2-fold in cultures with either of the immune splenocytes vs. cultures with naïve splenocytes, whereas MIP- $1 \alpha$ was the sole cytokine being suppressed in cultures with immune splenocytes (Table S1). It was further analyzed how the cytokine levels of the supernatants correlated to the degree of protection, i.e., growth inhibition. Also, the correlations between the levels of the 23 cytokines and IFN- $\gamma$ were analyzed. Growth inhibition was highly correlated $(P<0.01)$ to IFN$\gamma$, GM-CSF, IL-10, IL-13, MIP- $1 \alpha$, and RANTES and among these cytokines, GM-CSF, MIP-1 $\alpha$, and RANTES were highly correlated $(P<0.01)$ to IFN- $\gamma$ (Table 1$)$. In addition, IFN- $\gamma$ was highly correlated $(P<0.01)$ to IL-17, IL-6, IL-12p40, eotaxin, G-CSF, MCP-1, and MIP-1 $\beta$ (Table 1). From the 23 cytokines, 9 cytokines were chosen for use in subsequent analyses, since they all discriminated between levels in cultures with LVS- vs. $\Delta c l p B$-immunized splenocytes.

In summary, several of the cytokines, including IFN- $\gamma$, strongly correlated to growth inhibition. Superior containment of LVS bacteria was observed in cultures with $\Delta c l p B-$ vs. LVSimmune cells. The cytokine profile in the cultures with immune splenocytes reflected a Th1-type immune response.

\section{Characterization of Immune Splenocytes from the LVS-Infected Co-cultures}

To better understand the enhanced ability of the $\Delta c l p \mathrm{~B}$-immune splenocytes to control the replication of $F$. tularensis LVS, the percentage of IFN- $\gamma$-, TNF- $\alpha$-, and IL-17-producing T cells were quantified by FACS analysis. IL-17 has in several publications been found to correlate to protection against $F$. tularensis (Lin et al., 2009; Mahawar et al., 2013). Analysis was performed on non-adherent cells from the cultures after $72 \mathrm{~h}$ of incubation. There were higher percentages of $\mathrm{CD} 4^{+}$ and $\mathrm{CD}^{+} \mathrm{T}$ cells expressing IFN- $\gamma$ in cultures from the immunized groups $v s$. the naïve group $(P<0.05)$ and among the immunized groups, cultures with $\Delta c l p \mathrm{~B}$-immune splenocytes expressed higher percentages $(P<0.05)$ of IFN- $\gamma$-positive cells 

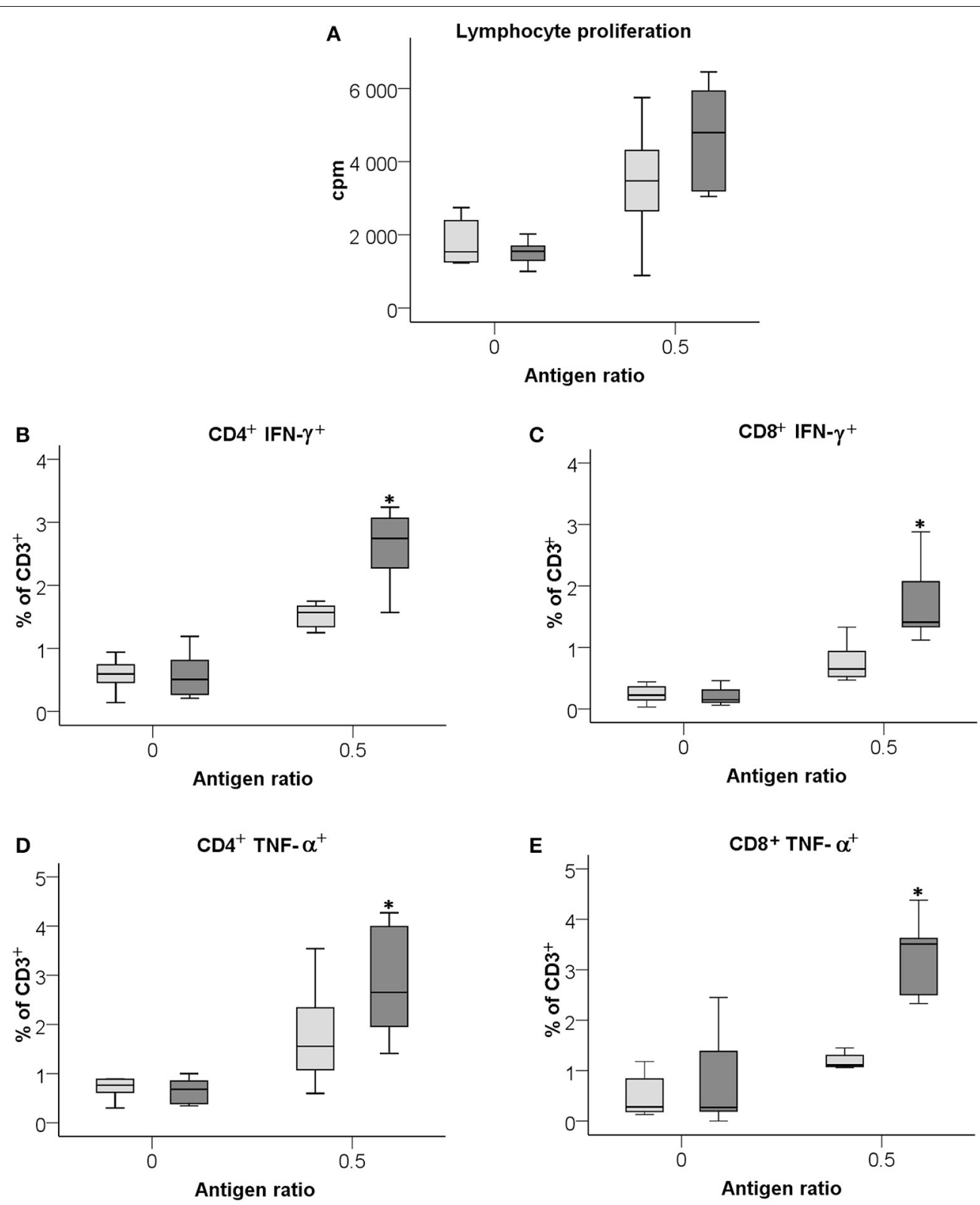

FIGURE 1 | Recall responses of LVS- (light gray boxes) or $\Delta$ clpB-immune (dark gray boxes) splenocytes analyzed with regard to lymphocyte proliferative responses (A), CD4 ${ }^{+}$T cells expressing IFN- $\gamma$ (B), CD8 ${ }^{+}$T-cell expressing IFN- $\gamma$ (C), CD4 ${ }^{+}$T cells expressing TNF- $\alpha$ (D), and CD8 ${ }^{+}$T-cell expressing TNF- $\alpha$ (E) Antigen ratio is expressed as number of killed bacteria/splenocyte. Results shown represent data from 3 experiments. Levels in cultures with naïve splenocytes were consistently $<1500 \mathrm{cpm}$ and showed no differences with or without antigen. Asterisks indicate $P<0.05$ according to Wilcoxon's signed rank test for the pair-wise comparisons between LVS- and $\Delta c / p B$-immune data for each antigen ratio.

among both the $\mathrm{CD}^{+}$and $\mathrm{CD}^{+}$subsets (Figure 3). Levels of cells expressing either IL-17 or TNF- $\alpha$ were similar between the immunized groups and the naïve group. Some studies have demonstrated that polyfunctional $\mathrm{T}$ cells demonstrate good correlation with host protection (Darrah et al., 2007; Derrick et al., 2011) and we identified such F. tularensis-specific T cells as part of the human immune response (Eneslätt et al., 2012). Also in the splenocyte-BMDM co-culture model, we observed the presence of polyfunctional $F$. tularensis-specific $\mathrm{T}$ cells, since in cultures with immune splenocytes, $\mathrm{CD} 4^{+}$and $\mathrm{CD} 8^{+}$
T cells expressing IFN- $\gamma$ and IL-17 or IFN- $\gamma$ and TNF- $\alpha$ were significantly increased compared to cultures with naïve cells $(P$ $<0.05)$. In all instances, the frequencies of the bifunctional $\mathrm{T}$ cells were significantly higher in cultures with $\Delta c l p \mathrm{~B}$-immune compared to LVS-immune splenocytes $(P<0.05)$ (Figure 3). Also, trifunctional $\mathrm{CD}^{+}$and $\mathrm{CD}^{+} \mathrm{T}$ cells were increased in cultures with immune splenocytes $(P<0.05)$ and, again, more frequent among $\Delta c l p \mathrm{~B}$-immune compared to LVS-immune splenocytes $(P<0.05$; Figure 3). Median fluorescence intensity (MFI) of the immune splenocytes was analyzed for IFN- $\gamma$, IL-17, 

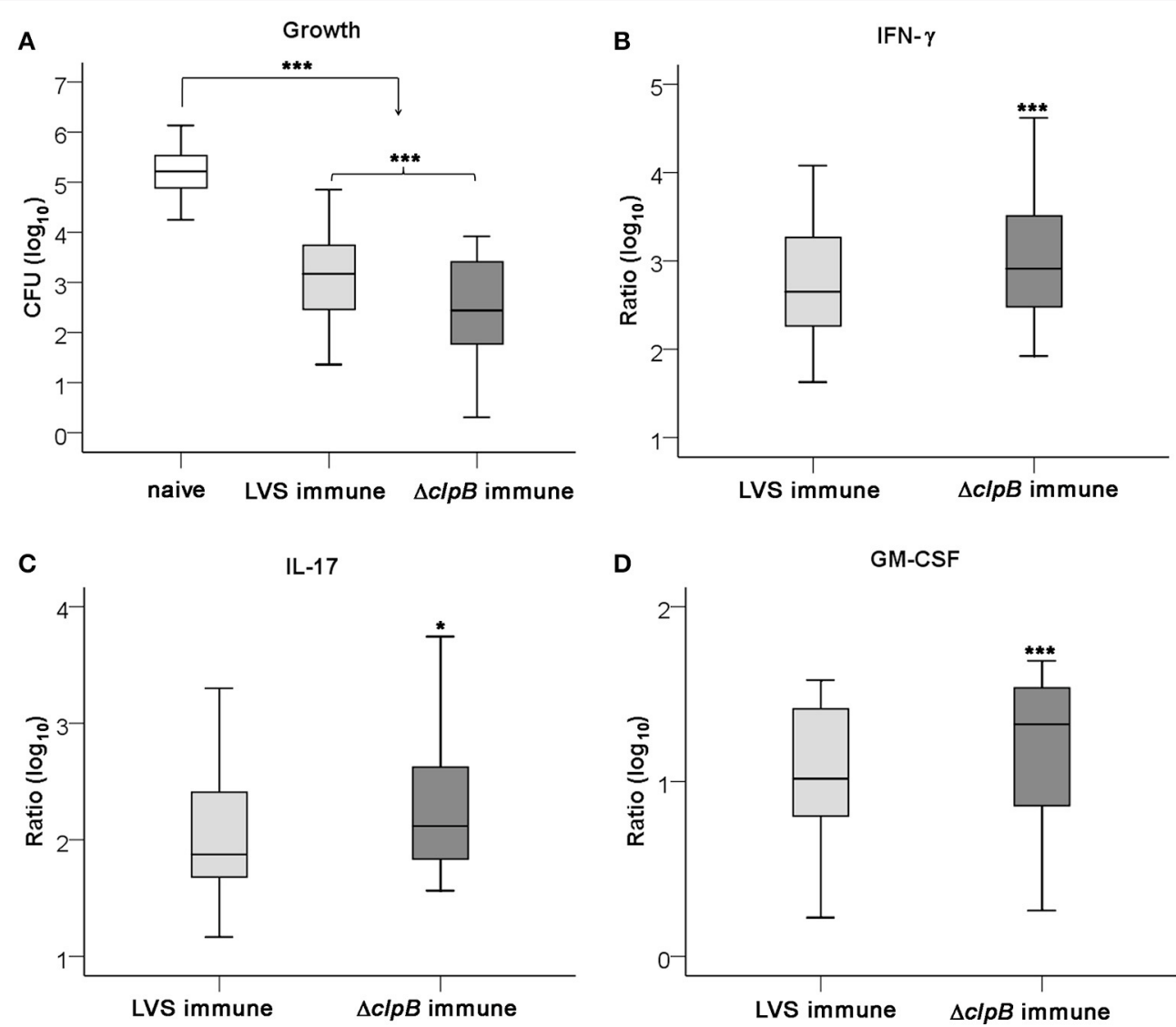

FIGURE 2 | Growth of $\boldsymbol{F}$. tularensis LVS and cytokine accumulation in the co-culture assay. Growth of LVS in cultures with naïve (white boxes), LVS-immune (light gray boxes) or $\Delta$ clpB-immune (dark gray boxes) splenocytes was determined after $72 \mathrm{~h}$ (A) and the accumulated levels of IFN- $\gamma$ (B), IL-17 (C), and GM-CSF (D) in these cultures. Results shown represent data from 16 experiments in graph A and 11 experiments in graphs (B-D). Growth was calculated as the log 10 CFU of cultures at $72 \mathrm{~h}$ subtracted with the $\log _{10} \mathrm{CFU}$ of cultures at $\mathrm{O}$ [median and IQ one and three $2.3(2.1,3.2)$ ]. In graphs (B-D), the values are expressed as log 10 fold-regulation of the indicated cytokine in relation to the concentration in cultures with naiive splenocytes. ${ }^{*} P<0.05,{ }^{* \star *} P<0.001$ according to Wilcoxon's signed rank test. In graphs (B-D), the significances indicate the pair-wise comparisons between LVS- and $\Delta c / p B$-immune data for each cytokine and in graph (A) for the indicated pair-wise comparisons.

and TNF- $\alpha$. Whereas, there were no significant differences for the latter two cytokines, $\Delta c l p B$-immune splenocytes demonstrated significantly higher MFI of IFN- $\gamma$ expression among the $\mathrm{CD} 4^{+} \mathrm{T}$ cell populations than did LVS-immune splenocytes, median levels were $2430[1890,7710]$ vs. $1210[670,3930](P<0.05)$.

Collectively, the data demonstrated that there were qualitative and quantitative differences between $\Delta c l p \mathrm{~B}$ - and LVS-immune splenocytes with regard to cytokine expression, since the $\Delta c l p B$-immune population contained higher frequencies of polyfunctional $\mathrm{CD}^{+}$and $\mathrm{CD}^{+} \mathrm{T}$ cells expressing IFN- $\gamma$ in combination with IL-17 and/or TNF- $\alpha$ and also higher MFI of the IFN- $\gamma$-expressing $\mathrm{CD} 4^{+} \mathrm{T}$ cells.

\section{Growth Inhibition of $\boldsymbol{F}$. tularensis SCHU S4 and Cytokine Production Conferred by LVS- or $\Delta c / p B-I m m u n e$ Splenocytes}

In agreement with our present findings, the splenocyte-BMDM co-culture assay has previously been demonstrated to mediate efficient control of LVS infection, but few studies have analyzed the requirements for the control of virulent $F$. tularensis strains (Mahawar et al., 2013; Griffin et al., 2015). Therefore, the same protocol as previously described for the LVS infection was used, but the highly virulent strain, SCHU S4, was instead used to infect the cultures. As was demonstrated for the LVS infection, $\Delta c l p \mathrm{~B}-$ immune splenocytes conferred superior control of SCHU S4 compared to LVS-immune splenocytes $(P<0.001$; Figure $4 \mathrm{~A})$. In fact, the LVS-immune cells did not restrict the growth of SCHU S4 to the same extent as of LVS, median growth was $4.2[3.1,4.4]$ vs. $3.2[2.4,3.8]$, respectively $(P<0.001$; Figures $2 \mathrm{~A}, \mathbf{4 A})$. In contrast, $\Delta c l p \mathrm{~B}$-immune Balb/c-derived splenocytes conferred as efficient growth inhibition of SCHU S4 as of LVS, median growth was $2.8[1.9,3.8]$ and $2.4[1.7,3.4] \log _{10} \mathrm{CFU}$, respectively $(P>0.05$; Figures 2A, 4A). The cytokine profile of SCHU S4infected cultures resembled that of LVS-infected cultures, but many of the cytokines were somewhat higher expressed in the cultures with SCHU S4 (Table S1). Thus, SCHU S4-infected cultures with $\Delta c l p \mathrm{~B}$-immune splenocytes contained higher levels 
of IFN- $\gamma$, GM-CSF, and IL-17 than the cultures with LVSimmune splenocytes $(P<0.01$ for IFN- $\gamma$ and GM-CSF and $P<$ 0.05 for IL-17: Figures 4B-D).

In summary, LVS-immune cells did not control SCHU S4 as well as it controlled LVS, whereas $\Delta c l p \mathrm{~B}$-immune cells showed superior control and controlled both strains to a similar extent. $\Delta c l p B$-immune cells induced higher levels of IFN- $\gamma$, IL-17, and GM-CSF than did LVS-immune cells.

\section{Nitric Oxide Production in Splenocyte-BMDM Co-Cultures}

Nitrite was measured in the supernatants of splenocyte-BMDM co-cultures infected with LVS and it was observed that the levels strongly correlated to growth inhibition (Spearman's rank correlation coefficient [rho] $=0.679, P<0.01$; Figure 5A). Nitrite levels were also highly correlated $(P<0.01)$ to levels of IFN- $\gamma$, GM-CSF, IL-6, IL-10, IL-12(p40), and RANTES (Table 1). $\Delta c l p \mathrm{~B}$-immune $\mathrm{Balb} / \mathrm{c}$-derived splenocytes stimulated higher nitrite production than did LVS-immune splenocytes, medians were $21 \mu \mathrm{M}[16,26]$ vs. $16 \mu \mathrm{M}[10,23]$, respectively $(P<0.05$; Figure 6). Also when SCHU S4 was the infecting agent, nitrite levels strongly correlated to growth inhibition (rho $=0.745, P$ $<0.001$; Figure 5B) and, again, cultures with $\Delta c l p \mathrm{~B}$-immune splenocytes displayed significantly higher levels of nitrite than did cultures with LVS-immune splenocytes, medians were $15[9,23]$ and $9[5,20] \mu \mathrm{M}$, respectively $(P<0.01$; Figure 6).

In summary, these results demonstrate that $\Delta c l p \mathrm{~B}$-immune splenocytes showed superior capacity compared to LVS-immune

TABLE 1 | Correlations between growth inhibition, nitrite levels, IFN- $\gamma$ levels and levels of other cytokines.

\begin{tabular}{|c|c|c|c|}
\hline & Growth inhibition $^{a}$ & IFN- $\gamma^{\mathbf{b}}$ & Nitrite ${ }^{c}$ \\
\hline Growth inhibition & 1.000 & $0.481^{\star *}$ & $0.679^{\star \star}$ \\
\hline $\mathrm{IFN}-\gamma$ & $0.481^{d^{\star \star}}$ & 1.000 & $0.525^{\star \star}$ \\
\hline Nitrite & $0.679^{\star \star}$ & $0.525^{\star \star}$ & 1.000 \\
\hline GM-CSF & $0.356^{\star \star}$ & $0.447^{\star \star}$ & $0.435^{\star \star}$ \\
\hline IL-10 & $0.499^{\star \star}$ & -0.007 & $0.560^{\star \star}$ \\
\hline $\mathrm{MIP}-1 \alpha$ & $0.576^{\star \star}$ & $0.727^{\star \star}$ & 0.238 \\
\hline $\mathrm{IL}-13$ & $0.672^{\star \star}$ & $0.566^{\star \star}$ & $0.440^{*}$ \\
\hline RANTES & $0.380^{\star \star}$ & $0.718^{\star \star}$ & $0.643^{\star \star}$ \\
\hline IL-17 & 0.145 & $0.697^{* \star}$ & 0.241 \\
\hline IL-6 & $0.281^{\star}$ & $0.898^{\star \star}$ & $0.416^{\star \star}$ \\
\hline IL-12(p40) & 0.252 & $0.607^{\star \star}$ & $0.488^{\star \star}$ \\
\hline Eotaxin & $0.353^{\star}$ & $0.441^{\star \star}$ & 0.186 \\
\hline G-CSF & 0.066 & $0.664^{* \star}$ & -0.244 \\
\hline MCP-1 & 0.228 & $0.713^{\star \star}$ & $-0.450^{*}$ \\
\hline MIP-1 $\beta$ & $0.307^{\star}$ & $0.495^{\star \star}$ & $0.342^{*}$ \\
\hline
\end{tabular}

a Growth inhibition of F. tularensis LVS in cultures with LVS-immune or $\Delta c / p B$-immune splenocytes vs. naive splenocytes in the in vitro assay after $72 \mathrm{~h}$.

${ }^{b}$ Levels of cytokines measured in the supernatants of the in vitro assay after $72 \mathrm{~h}$.

${ }^{c}$ Levels of nitrite measured in the supernatants of the in vitro assay after $72 \mathrm{~h}$.

a Spearman's rank correlation coefficient between indicated combination.

${ }^{\star} P<0.05 ;{ }^{* \star} P<0.01$. splenocytes to stimulate nitrite production in the splenocyteBMDM co-cultures and identify nitrite as a correlate of growth inhibition of both LVS and the highly virulent strain SCHU S4.

\section{Nitric Oxide-Dependent Growth Inhibition}

Since nitrite levels correlated to the growth inhibition of $F$. tularensis effectuated by the immune splenocytes, it was further investigated if there was a direct correlation between the growth inhibition of F. tularensis and NO by use of the competitive inhibitor of iNOS, NMMLA. This treatment significantly reduced the growth inhibition of LVS or SCHU S4 conferred by the immune Balb/c-derived splenocytes (Figures 7A,B) and also reduced the nitrite production to about $1 \mu \mathrm{M}$ compared to levels of at least $8.0 \mu \mathrm{M}$ in its absence (Figures 7C,D). Levels in cultures with naïve splenocytes were consistently below $1 \mu \mathrm{M}$.

The role of $\mathrm{NO}$ was further investigated by use of BMDM derived from iNOS ${ }^{-/-}$C57BL/6 mice. It should be noted that also these mice, like Balb/c mice, generate robust immunity after vaccination, since a majority survived an intradermal challenge of $1900 \mathrm{CFU}$ and all of 5 mice survived $190 \mathrm{CFU}$ after intradermal immunization with $\Delta c l p \mathrm{~B}$ (Table 2), whereas the lethal dose for naïve mice was one CFU. In contrast, all of LVS-immunized mice succumbed to a challenge of $19 \mathrm{CFU}$ within 22 days and maximal survival was even shorter when the challenge dose was 190 or 1900 CFU (Table 2). Thus, as for Balb/c mice (Conlan et al., 2010), immunization with $\Delta c l p B$ confers superior protection in C57BL/6 mice.

Similar to the splenocytes derived from Balb/cJ mice, C57BL/6-derived $\Delta c l p \mathrm{~B}$-immune splenocytes controlled growth of LVS and SCHU S4 better than did LVS-immune splenocytes, $P$ $<0.05$ and $P<0.01$, respectively (Figures 8A,B), and induced higher nitrite production in the cultures, $P<0.01$ and $P$ $<0.05$ for LVS- and SCHU S4-infected cultures, respectively

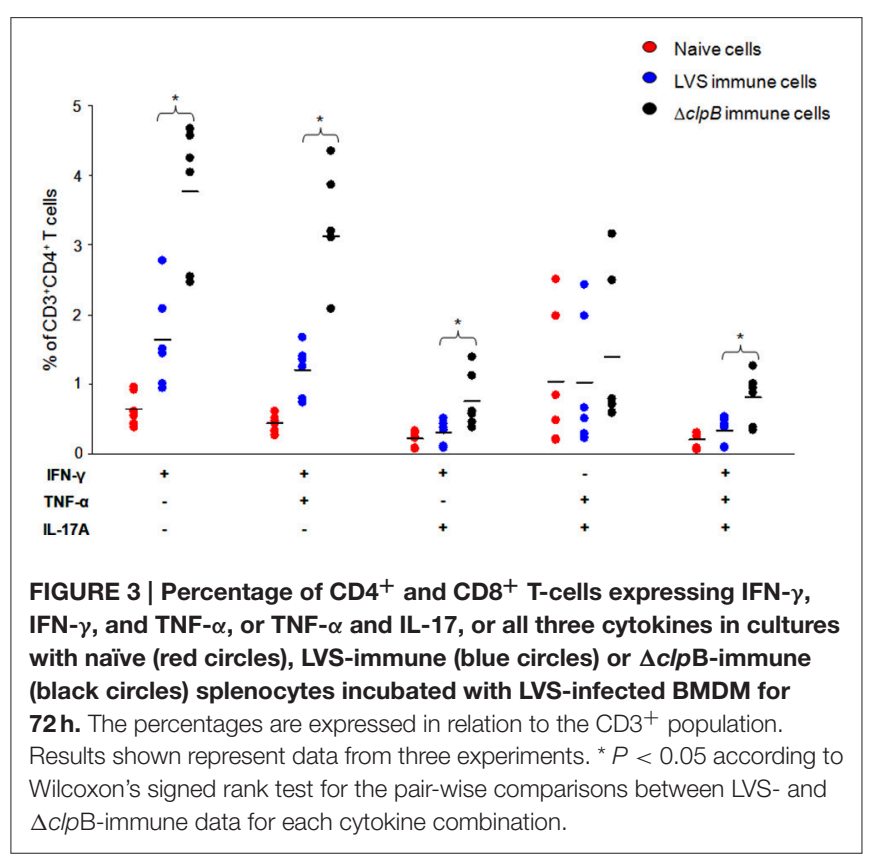



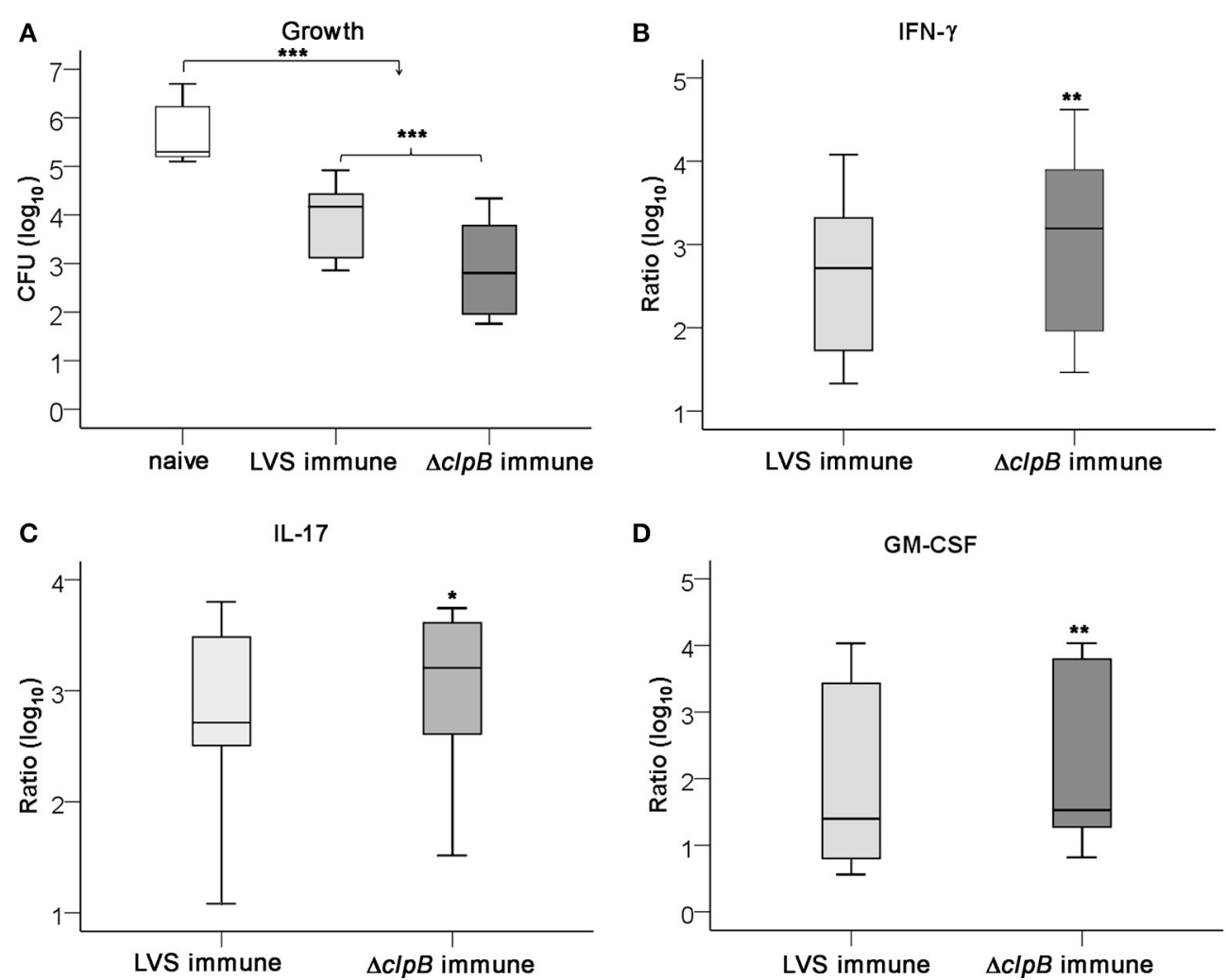

FIGURE 4 | Growth of $\boldsymbol{F}$. tularensis SCHU S4 and cytokine accumulation in the co-culture assay. Growth of SCHU S4 in cultures with naïve, LVS-immune or $\Delta c / p B$-immune splenocytes was determined after $72 \mathrm{~h}$ (A) and the accumulated levels of IFN- $\gamma$ (B), IL-17 (C), and GM-CSF (D) in these cultures. Results shown represent data from nine experiments. Growth was calculated as the CFU ( $\left.\log _{10}\right)$ of cultures at $72 \mathrm{~h}$ subtracted with the CFU (log 10$)$ of cultures at $0 \mathrm{~h}, 2.3$ [2.1, 3.0]. In graphs (B-D), the values are expressed as $\log _{10}$ fold-regulation of the indicated cytokine in relation to the concentration in cultures with naïve splenocytes. ${ }^{*} P<0.05$, ${ }^{\star \star} P<0.01,{ }^{\star \star \star} P<0.001$ according to Wilcoxon's signed rank test. In graphs (B-D), the significances indicate the pair-wise comparisons between LVS- and $\Delta c / p B$-immune data for each cytokine and in graph (A) for the indicated pair-wise comparisons.

(Figures 8C,D). Also in cultures with C57BL/6 cells, as in cultures with Balb/cJ cells, the nitrite levels were highly correlated to the growth inhibition of both LVS and SCHU S4 (rho = 0.745 and 0.771 , respectively, $P<0.01$ ). Further corroborating the role of $\mathrm{NO}$, the growth inhibition elicited by the immune splenocytes was significantly lower $(P<0.001)$ in cultures with iNOS $^{-/-}$BMDM compared to C57BL/6 BMDM, whether or not the cultures were infected with LVS or SCHU S4 (Figures 8A,B). However, even in the absence of iNOS-derived NO, LVSand $\Delta c l p B$-immune splenocytes still reduced the growth of LVS, median net growth were $4.3[3.9,4.7]$ and $3.5[3.4,3.7]$ $\log _{10}$ CFU, respectively, vs. $5.1[4.7,5.1]$ for naïve splenocytes; $\triangle c l p \mathrm{~B}$-immune splenocytes being significantly superior to LVSimmune splenocytes $(P<0.001$; Figure 8A). In contrast, growth inhibition of SCHU S4 in cultures with iNOS ${ }^{-/-}$BMDM was very minor or non-existent for both LVS- and $\Delta c l p B$-immune splenocytes (Figure 8B).

Collectively, the data obtained with C57BL/6-derived cells confirmed the results based on the Balb/c-derived cells, i.e., $\Delta c l p \mathrm{~B}$-immune splenocytes showed superior capacity compared to LVS-immune splenocytes to control the F. tularensis infection and the $\Delta c l p B$-immune splenocytes induced higher levels of nitrite production, which strongly correlated to growth inhibition. In addition, iNOS was found to be critically required for the growth inhibition of SCHU S4 and contributed significantly to the control of LVS.

\section{DISCUSSION}

It is well-established that protection against many intracellular pathogens is critically dependent on cell-mediated immunity, however, there are no methods validated to identify correlates of protection to these pathogens. In addition, for diseases like tularemia, which is infrequent in most parts of the world, the power of human clinical trials is unlikely to be sufficient to provide unambiguous data regarding the efficacy of vaccine candidates. To this end, the Animal Rule is an option for licensing of future tularemia vaccines and this option is most likely applicable to biodefense agents and sporadically occurring diseases, both of which are relevant to tularemia. Critically required in this regard is the identification of correlates of protection, i.e., a measurement of a biologically relevant function for and correlated to the degree of protection conferred in 


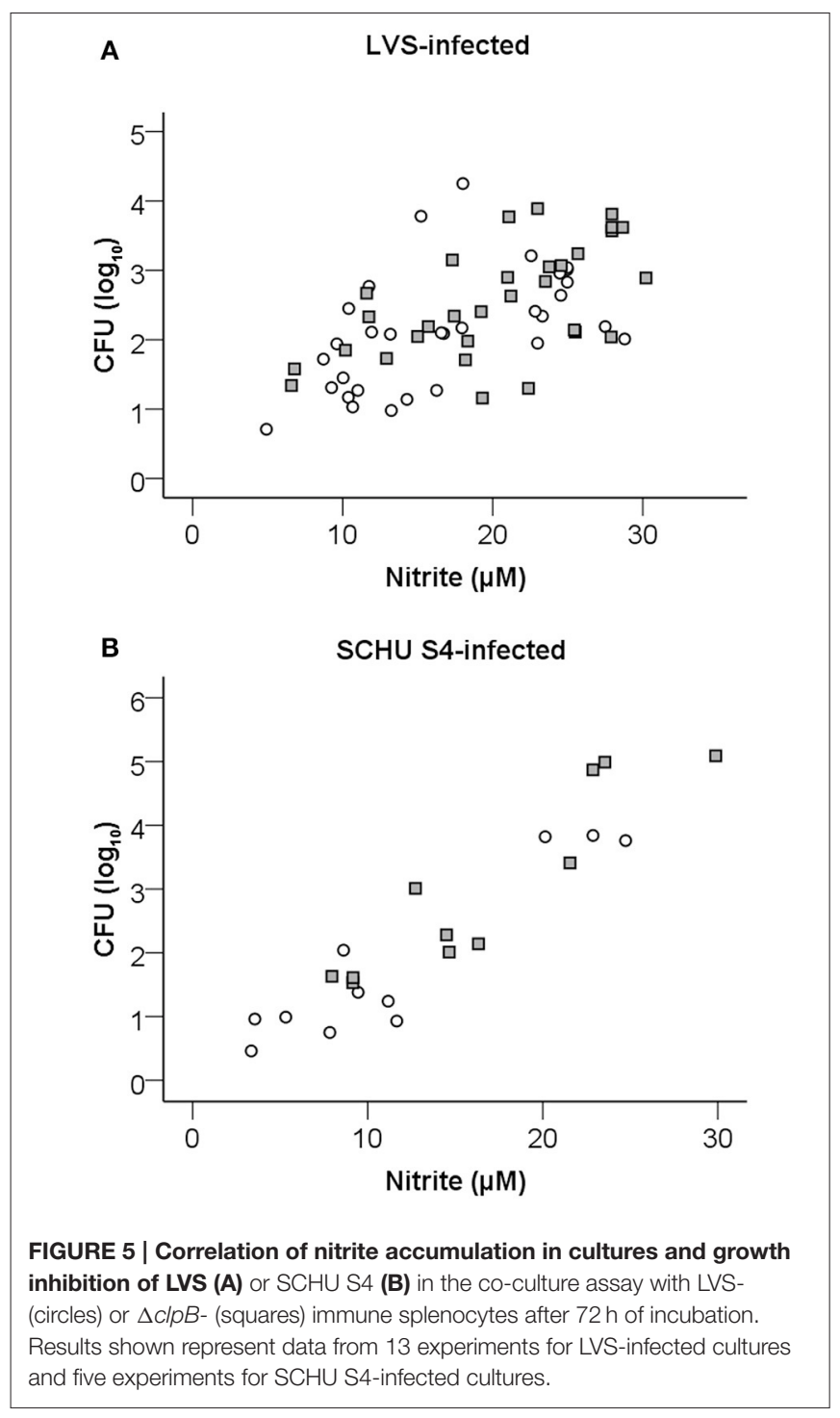

the animal model. For tularemia and other diseases caused by intracellular pathogens, e.g., tuberculosis, much focus has been on the role of IFN- $\gamma$ as a correlate of protection. Although important for the protection in vitro to Mycobacterium tuberculosis, levels of the cytokine have not shown good correlation to protection in various clinical and experimental models (Goldsack and Kirman, 2007; Mittrücker et al., 2007; Jeevan et al., 2009). With regard to tularemia, IFN- $\gamma$ appears to be necessary, although not sufficient for protection (Elkins et al., 2007; Conlan, 2011). Thus, there is a need to develop models to better define the correlates of protection against tularemia.

The use of the splenocyte-BMDM co-culture model demonstrates distinct advantages compared to commonly used animal models for tularemia, since the number of animals required will be far less and the mice that are immunized as a source of splenocytes receive a sublethal dose that results in few or no objective symptoms. Thus, the method leads to both distinct reduction of the number of animals required, as well as refinement, since the distress caused by the sublethal infection

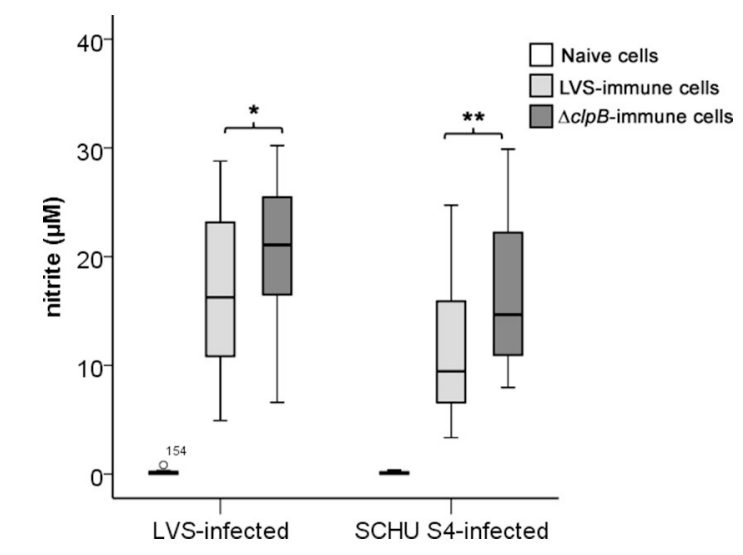

FIGURE 6 | Nitrite levels accumulated after $72 \mathrm{~h}$ in cultures with LVS- or SCHU S4-infected BMDM co-cultured with naïve (white boxes), LVS-immune (light gray boxes) or $\Delta c / p B$-immune (dark gray boxes) splenocytes. Results shown represent data from 13 experiments for LVS-infected cultures and five experiments for SCHU S4-infected cultures. * $P$ $<0.05,{ }^{\star \star} P<0.01$ according to Wilcoxon's signed rank test for the indicated pair-wise comparisons.

will be minimal. In addition, the use of the co-culture model allows for direct comparisons of correlates with those identified in human models. In fact, a human co-culture model, based on the use of adherent and non-adherent peripheral blood mononuclear leukocytes from LVS-vaccinated individuals or former tularemia patients, shows promise in this regard (Eneslätt et al, unpublished). Since it is likely that human challenge studies never will be performed in the future, the use of a human coculture model may be the only realistic way to identify correlates of immunity and protection. A limitation of co-culture models is there will be a selection of cell types included and the model may therefore not be representative for certain organ-specific immune responses in vivo. It should be noted that there are examples when organ-specific $F$. tularensis co-culture models have been utilized to overcome this limitation (De Pascalis et al., 2014).

Although previous studies based on splenocyte-BMDM coculture models have revealed much about the vaccine-mediated protection; both the prerequisites for protection against LVS as well as putative correlates of protection, there have been very few studies with virulent $F$. tularensis strains based on a co-culture system (Mahawar et al., 2013; Griffin et al., 2015).

The present study used the LVS strain as benchmark for F. tularensis vaccine efficacy. Vaccination with LVS effectively prevents laboratory-acquired infection, but studies on volunteers have revealed that it affords only marginal protection against aerosol infection (Saslaw et al., 1961a,b; Burke, 1977). It was hypothesized that it would be possible to generate a superior $F$. tularensis vaccine and we and others have generated a number of targeted mutants of SCHU S4 and their efficacy has been evaluated using in vivo models with virulent $F$. tularensis strains (Kadzhaev et al., 2009; Conlan et al., 2010; Shen et al., 2010; Ryden et al., 2012; Santiago et al., 2015). In particular one mutant, $\Delta c l p B$, was found to confer superior efficacy to LVS, as 

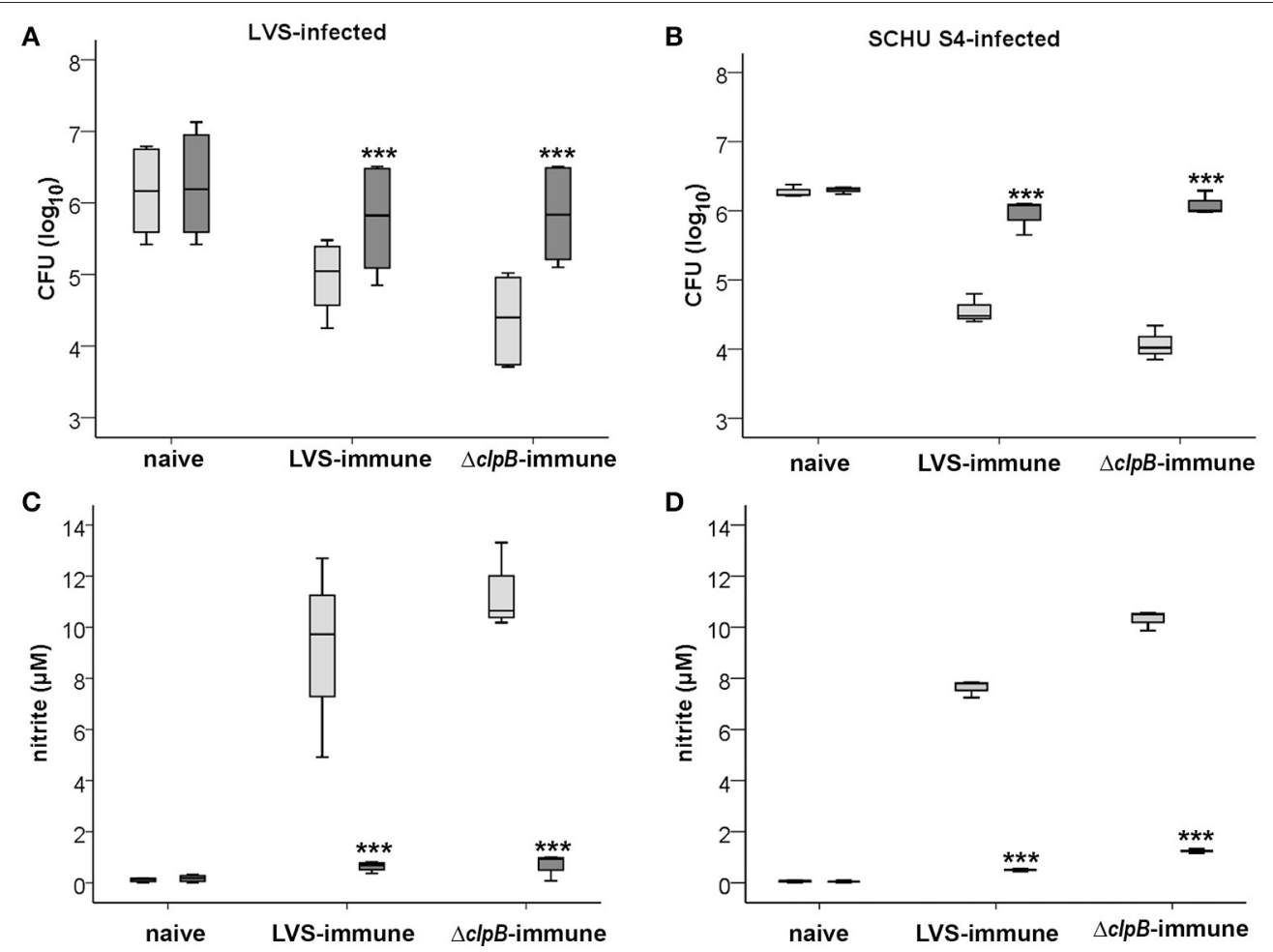

FIGURE 7 | Growth of $\boldsymbol{F}$. tularensis (A,B) and nitrite accumulation (C,D) in non-treated (light gray boxes) or NMMLA-treated (dark gray boxes) cultures. The data shown is from three experiments. ${ }^{\star \star \star} P<0.001$ according to Wilcoxon's signed rank test for the pair-wise comparisons between non-treated or NMMLA-treated cultures of each group.

TABLE 2 | Protective effect of LVS or $\triangle c / p B$ immunization of C57/BL6 mice against intradermal challenge with SCHU S4.

\begin{tabular}{llc}
\hline Immunization $^{\mathbf{a}}$ & Challenge dose $^{\mathbf{b}}$ & Time of survival (days) \\
\hline LVS & $19 \mathrm{CFU}$ & $7,8,9,11,22$ \\
$\Delta c / p B$ & $19 \mathrm{CFU}$ & $24,>33,>33,>33,>33$ \\
LVS & $190 \mathrm{CFU}$ & $7,8,12,12$ \\
$\Delta c / p B$ & $190 \mathrm{CFU}$ & $>33,>33,>33,>33,>33$ \\
LVS & $1900 \mathrm{CFU}$ & $7,8,8,9,17$ \\
$\Delta c / p B$ & $1900 \mathrm{CFU}$ & $17,22,33,33,33$ \\
None & $19 \mathrm{CFU}$ & $6,6,6,6,6$ \\
\hline
\end{tabular}

a Mice were intradermally immunized with 100,000 CFU of the LVS or the $\triangle$ ClpB strain. ${ }^{b}$ Mice were 6 weeks after immunization challenged intradermally with the indicated dose of SCHU S4.

demonstrated by survival after infection with SCHU S4 (Conlan et al., 2010). In agreement with the findings in both $\mathrm{Balb} / \mathrm{c}$ and C57BL/6 mice, our findings using the splenocyte-BMDM coculture method demonstrate that the $\Delta c l p B$-immune splenocytes were superior to LVS-immune splenocytes in several aspects, most notably that the $\Delta c l p \mathrm{~B}$-immune splenocytes conferred superior control of both LVS and SCHU S4 infection. In addition, LVS-immune splenocytes conferred significantly less control of SCHU S4 than LVS. Likewise, a previous study demonstrated that LVS-immune splenocytes controlled SCHU S4 infection only when pre-stimulated in vitro (Griffin et al., 2015).

Although previous studies based on various splenocyteBMDM co-culture models have revealed much about the vaccinemediated protection; both the prerequisites for protection against LVS as well as putative correlates of protection, there have been very few studies with virulent $F$. tularensis strains based on a co-culture system (Mahawar et al., 2013; Griffin et al., 2015) and, therefore, the present study provides important information regarding the protective mechanisms operative against the highly virulent SCHU S4 strain. In agreement with the previous studies (Mahawar et al., 2013; Griffin et al., 2015), our findings demonstrate a strong correlation between the ability of the vaccine strains to confer protection to virulent strains in vivo and their capability to efficiently prime the protective efficacy of the immune cells as measured by the splenocyteBMDM co-culture model. In addition, our study reveals that the quality of the immune responses elicited by LVS and $\Delta c l p \mathrm{~B}$ are similar, although the response elicited by the $\Delta c l p \mathrm{~B}$ vaccine is quantitatively more robust.

Multi-parameter flow cytometry has been used rather extensively to characterize memory $\mathrm{T}$ cells and the technique has enabled detailed descriptions of their phenotypic characteristics and functional abilities. In various experimental models, it has been argued that specific polyfunctional $\mathrm{T}$ cells demonstrate good correlation with host protection. While promising in the 

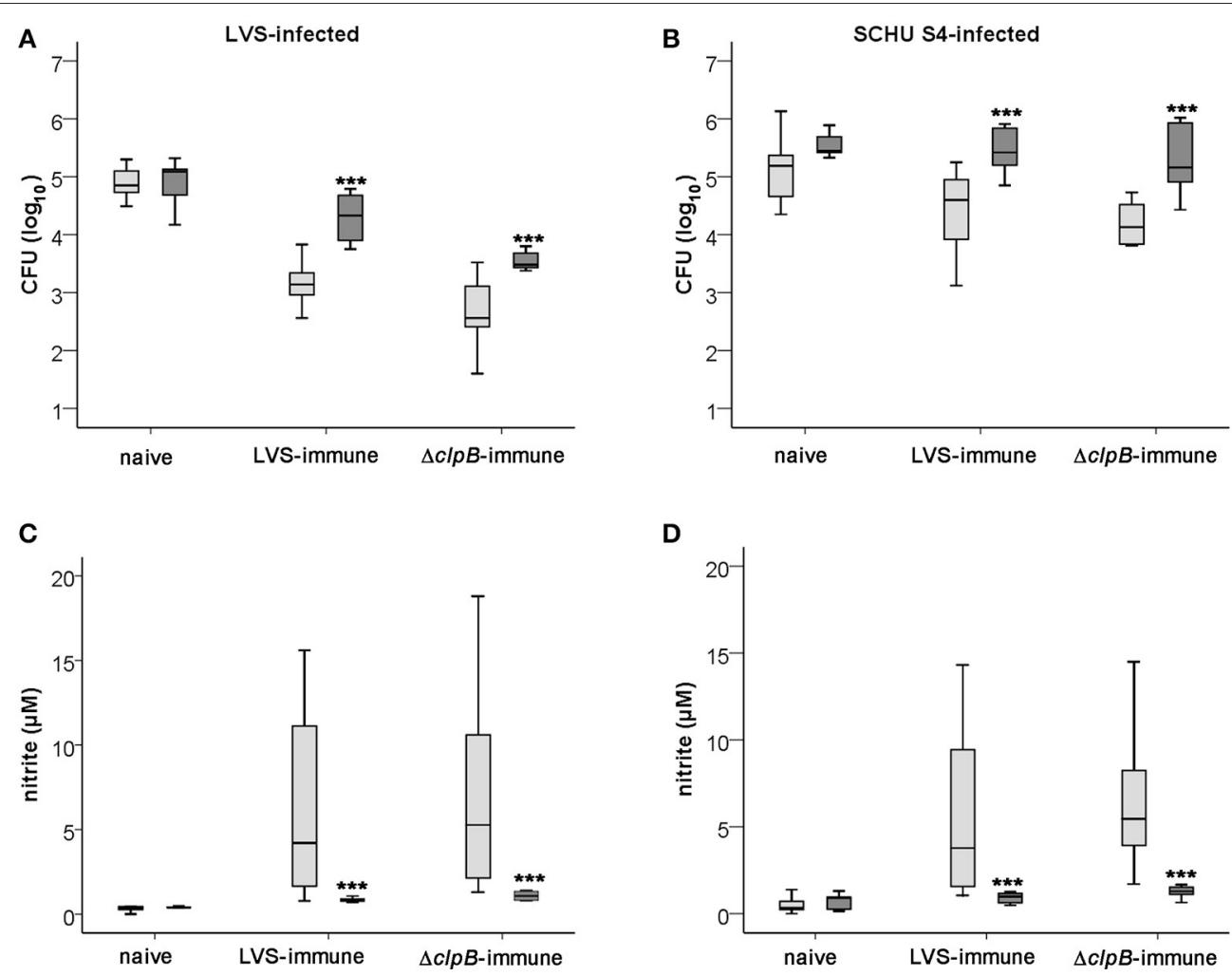

FIGURE 8 | Growth of $\boldsymbol{F}$. tularensis (A,B) and nitrite accumulation (C,D) in cultures with BMDM derived from C57BL/6 (light gray boxes) or iNOS ${ }^{-/-}$(dark gray boxes) mice cocultured with naive, LVS-immune or $\triangle c / p B$-immune splenocytes after $72 \mathrm{~h}$. (A) and (C) represent LVS-infected cultures and (B) and (D) SCHU S4-infected cultures. Results shown in graphs A and B represent data from 6 experiments for iNOS ${ }^{-/-}$BMDM and 8 experiments for C57BL/6 BMDM and in graphs $\mathrm{C}$ and $\mathrm{D}$ three experiments for $\mathrm{NOS}^{-/-}$BMDM and five experiments for C57BL/6 BMDM. ${ }^{* \star *} P<0.001$ according to Wilcoxon's signed rank test for the pair-wise comparisons between co-cultures with cells derived from C57BL/6 or iNOS ${ }^{-1-}$ mice of each group.

context of mouse models of Leishmania and certain tuberculosis vaccines (Darrah et al., 2007; Derrick et al., 2011), polyfunctional $\mathrm{T}$ cells have in other cases failed to demonstrate correlation to protection (Connor et al., 2010; Kagina et al., 2010; Harari et al., 2011). Human responses to killed $F$. tularensis antigens from the LVS or SCHU S4 strains have been characterized using multi-parameter flow cytometry and it was found that IFN- $\gamma$ and MIP- $1 \beta$ strongly discriminated between immune and naïve individuals (Eneslätt et al., 2012). Also in the present study, we found evidence that the immune cell populations contained polyfunctional T cells, expressing combinations of IFN- $\gamma$, TNF$\alpha$, and IL-17. Interestingly, the relative frequencies of all variants of polyfunctional $\mathrm{CD}^{+}$and $\mathrm{CD} 8^{+} \mathrm{T}$ cells expressing IFN$\gamma$ was consistently higher among $\Delta c l p B$ - than LVS-immune splenocytes.

Also in other important aspects, there were quantitative differences between the immune responses triggered by the $\Delta c l p \mathrm{~B}$ - $v s$. the LVS-immune splenocytes, since the $\Delta c l p \mathrm{~B}$ immune splenocytes demonstrated stronger proliferative responses and higher production of NO, IFN- $\gamma$, GM-CSF, and IL-17, all of which correlated to growth inhibition. Altogether, all of these properties of the $\Delta c l p \mathrm{~B}$-immune splenocytes, together with their superior ability to confer growth inhibition of SCHU
S4, help to explain the efficaciousness of $\Delta c l p B$. An interesting finding was that IFN- $\gamma$ was the most strongly induced cytokine after addition of immune splenocytes, whether or not they were derived from $\Delta c l p \mathrm{~B}$ - or LVS-immunized mice. Considering the critical role of IFN- $\gamma$ for many aspects of immunity to F. tularensis, the finding shows that the splenocyte-BMDM co-culture system used herein mirrors relevant aspects of other models of tularemia.

The effect of adding splenocytes derived from either C57BL/6 or Balb/cJ was also analyzed. In both instances, $\Delta c l p \mathrm{~B}$-immune splenocytes showed superior capacity compared to LVS-immune splenocytes to control the F. tularensis infection and also to induce production of higher levels of NO. This demonstrates that the distinct differences between $\Delta c l p B$-immune and LVSimmune splenocytes were not affected by the genetic background of the splenocytes.

It has been shown that control of LVS in a macrophageBMDM co-culture assay correlated with levels of nitrite (Bosio and Elkins, 2001; Elkins et al., 2009). Our results using the coculture method provided strong evidence for the critical role of NO for control also of the SCHU S4 infection as supported both by experiments using an inhibitor of iNOS, as well as iNOS-deficient BMDM. Although this may appear somewhat 
paradoxical, considering that NO plays a minor or insignificant role in vitro (Lindgren et al., 2005; Edwards et al., 2010), it is likely that NO production in the rather complex splenocyte-BMDM coculture model used herein confers additional effects compared to the model based on monocytic cells only. In further support of the important role of $\mathrm{NO}$, it exerts a critical function in vivo, since iNOS-deficient mice succumb even to the lowest inocula of an LVS infection (Lindgren et al., 2004).

The enhanced NO production in $\Delta c l p \mathrm{~B}$ cultures was likely dependent on the higher secretion of IFN- $\gamma$ and IL-17, and the higher frequency of polyfunctional $\mathrm{T}$ cells, expressing combinations of IFN-, TNF- $\alpha$, and IL-17, since these cytokines regulate iNOS. Regulation of iNOS is in many aspects distinct among species, but the critical roles of IFN- $\gamma$, TNF- $\alpha$, IL- $1 \beta$, and IL-17 to induce its expression appears to be conserved regardless of species (Pautz et al., 2010; Mühl et al., 2011). Many studies have demonstrated a critical role of NO in various murine infectious models, but there is also significant evidence supporting its role for control of human infectious diseases. One notable example is tuberculosis, the etiological agent of which is a facultative intracellular bacterium, as for tularemia. It has been observed that human M. tuberculosis-infected monocytes produce NO and this results in a bacteriostatic, and sometimes even bactericidal effect (Landes et al., 2015). Further corroborating an important role of $\mathrm{NO}$, it has been observed that iNOS expression is increased in human granulomas (Mattila et al., 2013). Malaria is another important human disease where there is evidence for a critical role of $\mathrm{NO}$, since endogenous production of asymmetrical dimethylarginine, an iNOS inhibitor, strongly correlated to mortality (Yeo et al., 2010). Collectively, such findings suggest that NO could have an important role in other human diseases, such as tularemia, and, therefore, that the findings regarding the

\section{REFERENCES}

Bosio, C. M., and Elkins, K. L. (2001). Susceptibility to secondary Francisella tularensis live vaccine strain infection in B-cell-deficient mice is associated with neutrophilia but not with defects in specific T-cell-mediated immunity. Infect. Immun. 69, 194-203. doi: 10.1128/IAI.69.1.194-203.2001

Burke, D. S. (1977). Immunization against tularemia: analysis of the effectiveness of live Francisella tularensis vaccine in prevention of laboratory-acquired tularemia. J. Infect. Dis. 135, 55-60. doi: 10.1093/infdis/135.1.55

Collazo, C. M., Meierovics, A. I., De Pascalis, R., Wu, T. H., Lyons, C. R., and Elkins, K. L. (2009). T cells from lungs and livers of Francisella tularensis immune mice control the growth of intracellular bacteria. Infect. Immun. 77, 2010-2021. doi: 10.1128/IAI.01322-08

Conlan, J. W. (2011). Tularemia vaccines: recent developments and remaining hurdles. Future Microbiol. 6, 391-405. doi: 10.2217/fmb.11.22

Conlan, J. W., Shen, H., Golovliov, I., Zingmark, C., Oyston, P. C., Chen, W., et al. (2010). Differential ability of novel attenuated targeted deletion mutants of Francisella tularensis subspecies tularensis strain SCHU S4 to protect mice against aerosol challenge with virulent bacteria: effects of host background and route of immunization. Vaccine 28, 1824-1831. doi: 10.1016/j.vaccine.2009.12.001

Connor, L. M., Harvie, M. C., Rich, F. J., Quinn, K. M., Brinkmann, V., Le Gros, G., et al. (2010). A key role for lung-resident memory lymphocytes in protective immune responses after BCG vaccination. Eur. J. Immunol. 40, 2482-2492. doi: 10.1002/eji.200940279 superior protection conferred by $\Delta c l p \mathrm{~B}$ in the mouse model also will have relevance for the human situation.

Overall, our results provide new information as to why $\Delta c l p \mathrm{~B}$ is superior to LVS as a vaccine for protection against highly virulent $F$. tularensis. The information is critical for the understanding of protective mechanisms and thereby for important for licensing of future vaccines against this potent pathogen.

\section{AUTHOR CONTRIBUTIONS}

Conceived and designed the experiments: IG, HL, KE, AS, Performed the experiments: IG, HL, KE, AM, WC, Analyzed the data: IG; HL, KE, WC, AS, Contributed reagents/materials/analysis tools: AM, TH. Wrote the paper: IG, HL, TH, AS.

\section{FUNDING}

Grant support was obtained from the Swedish Medical Research Council (K2012-3469 and K2013-8621), Västerbottens läns landsting (Spjutspetsmedel, VLL-582571, Centrala ALF medel, VLL-463691), the Medical Faculty, Umea University, Umeå, Sweden, and from the ERC (ERC starting grant 311542 to $\mathrm{TH})$. The work was performed in part at the Umeå Centre for Microbial Research (UCMR).

\section{SUPPLEMENTARY MATERIAL}

The Supplementary Material for this article can be found online at: http://journal.frontiersin.org/article/10.3389/fcimb. 2016.00152/full\#supplementary-material

Cowley, S. C., and Elkins, K. L. (2003). Multiple T cell subsets control Francisella tularensis LVS intracellular growth without stimulation through macrophage interferon gamma receptors. J. Exp. Med. 198, 379-389. doi: $10.1084 /$ jem. 20030687

Cowley, S. C., and Elkins, K. L. (2011). Immunity to Francisella. Front. Microbiol. 2:26. doi: $10.3389 /$ fmicb. 2011.00026

Cowley, S. C., Hamilton, E., Frelinger, J. A., Su, J., Forman, J., and Elkins, K. L. (2005). CD4-CD8- T cells control intracellular bacterial infections both in vitro and in vivo. J. Exp. Med. 202, 309-319. doi: 10.1084/jem.20050569

Darrah, P. A., Patel, D. T., De Luca, P. M., Lindsay, R. W., Davey, D. F., Flynn, B. J., et al. (2007). Multifunctional TH1 cells define a correlate of vaccine-mediated protection against Leishmania major. Nat. Med. 13, 843-850. doi: $10.1038 / \mathrm{nm} 1592$

De Pascalis, R., Chou, A. Y., Bosio, C. M., Huang, C. Y., Follmann, D. A., and Elkins, K. L. (2012). Development of functional and molecular correlates of vaccine-induced protection for a model intracellular pathogen, Francisella tularensis LVS. PLoS Pathog. 8:e1002494. doi: 10.1371/journal.ppat.1002494

De Pascalis, R., Chou, A. Y., Ryden, P., Kennett, N. J., Sjöstedt, A., and Elkins, K. L. (2014). Models derived from in vitro analyses of spleen, liver, and lung leukocyte functions predict vaccine efficacy against the Francisella tularensis Live Vaccine Strain (LVS). MBio 5:e00936. doi: 10.1128/mbio.00936-13

De Pascalis, R., Taylor, B. C., and Elkins, K. L. (2008). Diverse myeloid and lymphoid cell subpopulations produce gamma interferon during early innate immune responses to Francisella tularensis live vaccine strain. Infect. Immun. 76, 4311-4321. doi: 10.1128/IAI.00514-08 
Derrick, S. C., Yabe, I. M., Yang, A., and Morris, S. L. (2011). Vaccineinduced anti-tuberculosis protective immunity in mice correlates with the magnitude and quality of multifunctional CD4 T cells. Vaccine 29, 2902-2909. doi: 10.1016/j.vaccine.2011.02.010

Edwards, J. A., Rockx-Brouwer, D., Nair, V., and Celli, J. (2010). Restricted cytosolic growth of Francisella tularensis subsp. tularensis by IFN-gamma activation of macrophages. Microbiology 156, 327-339. doi: $10.1099 /$ mic. $0.031716-0$

Elkins, K. L., Colombini, S. M., Krieg, A. M., and De Pascalis, R. (2009). NK cells activated in vivo by bacterial DNA control the intracellular growth of Francisella tularensis LVS. Microbes Infect. 11, 49-56. doi: 10.1016/j.micinf.2008.10.005

Elkins, K. L., Cowley, S. C., and Bosio, C. M. (2007). Innate and adaptive immunity to Francisella. Ann. N.Y. Acad. Sci. 1105, 284-324. doi: 10.1196/annals.1409.014

Elkins, K. L., Cowley, S. C., and Conlan, J. W. (2011). Measurement of macrophage-mediated killing of intracellular bacteria, including Francisella and mycobacteria. Curr. Protoc. Immunol. Chapter 14, Unit14 25. doi: 10.1002/0471142735.im1425s93

Eneslätt, K., Normark, M., Björk, R., Rietz, C., Zingmark, C., Wolfraim, L. A., et al. (2012). Signatures of T cells as correlates of immunity to Francisella tularensis. PLoS ONE 7:e32367. doi: 10.1371/journal.pone.0032367

Eneslätt, K., Rietz, C., Rydén, P., Stöven, S., House, R. V., Wolfraim, L. A., et al. (2011). Persistence of cell-mediated immunity three decades after vaccination with the live vaccine strain of Francisella tularensis. Eur. J. Immunol. 41, 974-980. doi: 10.1002/eji.201040923

Ericsson, M., Sandström, G., Sjöstedt, A., and Tärnvik, A. (1994). Persistence of cell-mediated immunity and decline of humoral immunity to the intracellular bacterium Francisella tularensis 25 years after natural infection. J. Infect. Dis. 170, 110-114. doi: 10.1093/infdis/ 170.1.110

Giovannoni, G., Land, J. M., Keir, G., Thompson, E. J., and Heales, S. J. (1997). Adaptation of the nitrate reductase and Griess reaction methods for the measurement of serum nitrate plus nitrite levels. Ann. Clin. Biochem. 34, 193-198. doi: 10.1177/000456329703400212

Goldsack, L., and Kirman, J. R. (2007). Half-truths and selective memory: interferon gamma, CD4 $\mathrm{T}$ cells and protective memory against tuberculosis. Tuberculosis (Edinb) 87, 465-473. doi: 10.1016/j.tube.2007. 07.001

Golovliov, I., Baranov, V., Krocova, Z., Kovarova, H., and Sjöstedt, A. (2003). An attenuated strain of the facultative intracellular bacterium Francisella tularensis can escape the phagosome of monocytic cells. Infect. Immun. 71, 5940-5950. doi: 10.1128/IAI.71.10.5940-5950.2003

Griffin, A. J., Crane, D. D., Wehrly, T. D., and Bosio, C. M. (2015). Successful protection against tularemia in C57BL/6 mice is correlated with expansion of Francisella tularensis-specific effector T cells. Clin. Vaccine Immunol. 22, 119-128. doi: 10.1128/CVI.00648-14

Harari, A., Rozot, V., Bellutti Enders, F., Perreau, M., Stalder, J. M., Nicod, L. P., et al. (2011). Dominant TNF-alpha Mycobacterium tuberculosis-specific CD4 $\mathrm{T}$ cell responses discriminate between latent infection and active disease. Nat. Med. 17, 372-376. doi: 10.1038/ nm.2299

Jeevan, A., Bonilla, D. L., and McMurray, D. N. (2009). Expression of interferon-gamma and tumour necrosis factor-alpha messenger RNA does not correlate with protection in guinea pigs challenged with virulent Mycobacterium tuberculosis by the respiratory route. Immunology 128, e296e305. doi: 10.1111/j.1365-2567.2008.02962.x

Kadzhaev, K., Zingmark, C., Golovliov, I., Bolanowski, M., Shen, H., Conlan, W., et al. (2009). Identification of genes contributing to the virulence of Francisella tularensis SCHU S4 in a mouse intradermal infection model. PLoS ONE 4:e5463. doi: 10.1371/journal.pone.0005463

Kagina, B. M., Abel, B., Scriba, T. J., Hughes, E. J., Keyser, A., Soares, A., et al. (2010). Specific T cell frequency and cytokine expression profile do not correlate with protection against tuberculosis after bacillus Calmette-Guerin vaccination of newborns. Am. J. Respir. Crit. Care Med. 182, 1073-1079. doi: 10.1164/rccm.201003-0334OC

Kurtz, S. L., Foreman, O., Bosio, C. M., Anver, M. R., and Elkins, K. L. (2013). Interleukin-6 is essential for primary resistance to Francisella tularensis live vaccine strain infection. Infect. Immun. 81, 585-597. doi: 10.1128/IAI.01249-12
Landes, M. B., Rajaram, M. V., Nguyen, H., and Schlesinger, L. S. (2015) Role for NOD2 in Mycobacterium tuberculosis-induced iNOS expression and NO production in human macrophages. J. Leukoc. Biol. 97, 1111-1119. doi: 10.1189/jlb.3A1114-557R

Lin, Y., Ritchea, S., Logar, A., Slight, S., Messmer, M., Rangel-Moreno, J., et al. (2009). Interleukin-17 is required for $\mathrm{T}$ helper 1 cell immunity and host resistance to the intracellular pathogen Francisella tularensis. Immunity 31, 799-810. doi: 10.1016/j.immuni.2009.08.025

Lindgren, H., Stenman, L., Tarnvik, A., and Sjöstedt, A. (2005). The contribution of reactive nitrogen and oxygen species to the killing of Francisella tularensis LVS by murine macrophages. Microbes Infect. 7, 467-475. doi: 10.1016/j.micinf.2004.11.020

Lindgren, H., Stenmark, S., Chen, W., Tärnvik, A., and Sjöstedt, A. (2004). Distinct roles of reactive nitrogen and oxygen species to control infection with the facultative intracellular bacterium Francisella tularensis. Infect. Immun. 72, 7172-7182. doi: 10.1128/IAI.72.12.7172-7182.2004

Mahawar, M., Rabadi, S. M., Banik, S., Catlett, S. V., Metzger, D. W., Malik, M., et al. (2013). Identification of a live attenuated vaccine candidate for tularemia prophylaxis. PLoS ONE 8:e61539. doi: 10.1371/journal.pone.0061539

Mattila, J. T., Ojo, O. O., Kepka-Lenhart, D., Marino, S., Kim, J. H., Eum, S. Y., et al. (2013). Microenvironments in tuberculous granulomas are delineated by distinct populations of macrophage subsets and expression of nitric oxide synthase and arginase isoforms. J. Immunol. 191, 773-784. doi: 10.4049/jimmunol.1300113

Melillo, A. A., Foreman, O., Bosio, C. M., and Elkins, K. L. (2014). T-bet regulates immunity to Francisella tularensis live vaccine strain infection, particularly in lungs. Infect. Immun. 82, 1477-1490. doi: 10.1128/IAI. 01545-13

Melillo, A. A., Foreman, O., and Elkins, K. L. (2013). IL-12Rbeta2 is critical for survival of primary Francisella tularensis LVS infection. J. Leukoc. Biol. 93, 657-667. doi: 10.1189/jlb.1012485

Mittrücker, H. W., Steinhoff, U., Köhler, A., Krause, M., Lazar, D., Mex, P., et al. (2007). Poor correlation between BCG vaccination-induced T cell responses and protection against tuberculosis. Proc. Natl. Acad. Sci. U.S.A. 104, 12434-12439. doi: 10.1073/pnas.0703510104

Mühl, H., Bachmann, M., and Pfeilschifter, J. (2011). Inducible NO synthase and antibacterial host defence in times of Th17/Th22/T22 immunity. Cell. Microbiol. 13, 340-348. doi: 10.1111/j.1462-5822.2010. 01559.x

Pautz, A., Art, J., Hahn, S., Nowag, S., Voss, C., and Kleinert, H. (2010). Regulation of the expression of inducible nitric oxide synthase. Nitric Oxide 23, 75-93. doi: 10.1016/j.niox.2010.04.007

Ryden, P., Twine, S., Shen, H., Harris, G., Chen, W., Sjöstedt, A., et al. (2012). Correlates of protection following vaccination of mice with gene deletion mutants of Francisella tularensis subspecies tularensis strain, SCHU S4 that elicit varying degrees of immunity to systemic and respiratory challenge with wild-type bacteria. Mol. Immunol. 54, 58-67. doi: 10.1016/j.molimm.2012.10.043

Santiago, A. E., Mann, B. J., Qin, A., Cunningham, A. L., Cole, L. E., Grassel, C., et al. (2015). Characterization of Francisella tularensis Schu S4 defined mutants as live-attenuated vaccine candidates. Pathog. Dis. 73:ftv036. doi: 10.1093/femspd/ftv036

Saslaw, S., Eigelsbach, H. T., Prior, J. A., Wilson, H. E., and Carhart, S. (1961a). Tularemia vaccine study. II. Respiratory challenge. Arch. Intern. Med. 107, 702-714. doi: 10.1001/archinte.1961.03620050068007

Saslaw, S., Eigelsbach, H. T., Wilson, H. E., Prior, J. A., and Carhart, S. (1961b). Tularemia vaccine study. I. Intracutaneous challenge. Arch. Intern. Med. 107, 689-701. doi: 10.1001/archinte.1961.03620050055006

Shen, H., Harris, G., Chen, W., Sjöstedt, A., Ryden, P., and Conlan, W. (2010). Molecular immune responses to aerosol challenge with Francisella tularensis in mice inoculated with live vaccine candidates of varying efficacy. PLoS ONE 5:e13349. doi: 10.1371/journal.pone.0013349

Sjöstedt, A. (2007). Tularemia: history, epidemiology, pathogen physiology, and clinical manifestations. Ann. N.Y. Acad. Sci. 1105, 1-29. doi: 10.1196/annals.1409.009

Snoy, P. (2010). Establishing efficacy of human products using animals: the US Food and Drug Administration's "animal rule." Vet. Pathol. 47, 774-778. doi: 10.1177/0300985810372506 
US. Food and Drug Administration. (2015). FDA Approves Vaccine for use After Known or Suspected Anthrax Exposure. Available online at: http://www.fda.gov/ NewsEvents/Newsroom/PressAnnouncements/ucm474027.htm

Yeo, T. W., Lampah, D. A., Tjitra, E., Gitawati, R., Darcy, C. J., Jones, C., et al. (2010). Increased asymmetric dimethylarginine in severe falciparum malaria: association with impaired nitric oxide bioavailability and fatal outcome. PLoS Pathog. 6:e1000868. doi: 10.1371/annotation/e49842d3-ea72-45a4-93d4-2f494baee962

Conflict of Interest Statement: The authors declare that the research was conducted in the absence of any commercial or financial relationships that could be construed as a potential conflict of interest.
The reviewer TEP and handling Editor declared their shared affiliation and the handling Editor states that the process nevertheless met the standards of a fair and objective review.

Copyright (c) 2016 Golovliov, Lindgren, Eneslätt, Conlan, Mosnier, Henry and Sjöstedt. This is an open-access article distributed under the terms of the Creative Commons Attribution License (CC BY). The use, distribution or reproduction in other forums is permitted, provided the original author(s) or licensor are credited and that the original publication in this journal is cited, in accordance with accepted academic practice. No use, distribution or reproduction is permitted which does not comply with these terms. 